IJMMS 2003:4, 251-262

PII. S0161171203013097

http://ijmms.hindawi.com

(c) Hindawi Publishing Corp.

\title{
ON KRULL'S INTERSECTION THEOREM OF FUZZY IDEALS
}

\author{
V. MURALI and B. B. MAKAMBA
}

Received 11 May 2001

\begin{abstract}
We deal with Krull's intersection theorem on the ideals of a commutative Noetherian ring in the fuzzy setting. We first characterise products of finitely generated fuzzy ideals in terms of fuzzy points. Then, we study the question of uniqueness and existence of primary decompositions of fuzzy ideals. Finally, we use such decompositions and a form of Nakayama's lemma to prove the Krull intersection theorem. Fuzzy-points method on finitely generated fuzzy ideals plays a central role in the proofs.
\end{abstract}

2000 Mathematics Subject Classification: 13A15, 03E72, $13 \mathrm{C} 12$.

1. Introduction. After the first paper on fuzzy groups in 1971 by Rosenfeld, a decade passed before researchers started to look more closely into the notions extending fuzzy subsets to groups, rings, vector spaces, and other algebraic objects, $[2,3,8,9,10]$. There were attempts to unify these studies in one coherent way in the form of modules, $[4,5,6]$. The notion of prime ideal was generalized to fuzzy prime ideals of a ring, and thus initiating the study of radicals and primary ideals in the fuzzy case by Malik and Mordeson $[7,8,9,10,11]$. In this paper, we study the concepts of primary decompositions of fuzzy ideals and the radicals of such ideals over a commutative ring. Using such decompositions and a form of Nakayama's lemma, we prove Krull's intersection theorem on fuzzy ideals. We cleverly use the idea of fuzzy points belonging to fuzzy subsets to work out a number of proofs, creating a new way of extending ideas (see, e.g., Proposition 3.3, Theorems 3.5 and 3.11, Proposition 5.2, and so on). (Even though these results are known in the literature, our proofs are new and are based on the fuzzy-point concept.)

We briefly describe the framework of the paper. In Section 2, we introduce notions from fuzzy set theory such as fuzzy ideals, fuzzy points, sums and products on fuzzy ideals, and finitely generated fuzzy ideals. In Section 3, we recall definitions of prime, primary ideals, and their radicals from [11]. Then, we state and prove two uniqueness theorems of primary decompositions. In Section 4, we introduce irreducible fuzzy ideals to establish the existence theorem of primary decomposition. Section 5 deals with the Krull intersection theorem, namely $\bigwedge_{n=1}^{\infty} \mu^{n}=0$ under suitable conditions on $\mu$.

2. Preliminaries. In this paper, $R$ denotes a commutative Noetherian ring with unity 1 . In general, we are interested in the intersections of ideals in $R$. 
Nakayama's lemma states that if $M$ is a finitely generated module over $R$ and if $I$ is an ideal contained in the Jacobson radical of $R$ with $M=I M$, then $M=0$. A consequence of this fact is that if $I$ is an ideal of a commutative Noetherian ring $R$ contained in the Jacobson radical of $R$, then $\cap_{n=1}^{\infty} I^{n}=0$. In the literature, this is one of the forms in which Krull's intersection theorem is stated. In the following, we are interested in translating these results in the fuzzy set theory setting. Firstly, we summarise the basic theory of fuzzy ideals in order to set the foundation leading up to decomposition theorems of fuzzy ideals. We use $[0,1]$, the real unit interval, as a chain with the usual ordering in which $\wedge$ stands for infimum (or intersection) and $\vee$ stands for supremum (or union). If $R$ is treated as a set, then any mapping $\mu: R \rightarrow[0,1]$ is known as a fuzzy subset of $R$. The pointwise ordering of the power set $I^{R}$ induces the notions of containment, intersection, and union among the fuzzy subsets of $R$ in a natural way, viz. $\mu \leq v$ if and only if $\mu(r) \leq v(r)$ for all $r \in R$. In particular, the top element is $\chi_{R}$, which we denote by $R$. The bottom element is $\chi_{0}$, which we denote by 0 . A fuzzy subgroup of $R$ is a mapping $\mu: R \rightarrow[0,1]$ such that $\mu(r)=\mu(-r)$ for all $r \in R$ and $\mu(r+s) \geq \mu(r) \wedge \mu(s)$ for all $r, s \in R$. A fuzzy subset $\mu: R \rightarrow[0,1]$ is a fuzzy ideal of $R$ if (i) $\mu$ is a fuzzy subgroup of $R$ and (ii), for each pair $r, s \in R$, we have $\mu(r s) \geq \mu(r) \vee \mu(s)$. When $R$ is a field, (ii) becomes $\mu(r s)=\mu(s)$ for all $r \in R \backslash\{0\}, s \in R$. Suppose that $M$ is a module over $R$ and $\mu$ is a fuzzy subgroup of $M$; then, $\mu$ is a fuzzy submodule of $M$ if, for each $r \in R$ and $m \in M$, we have $\mu(r m) \geq \mu(m)$. Let $t \in[0, \mu(0)]$ where the 0 in $\mu(0)$ denotes the additive identity in $R$, and $\mu$ is a fuzzy ideal of $R$. The subset $\mu^{t}=\{r \in R: \mu(r) \geq t\}$ is called the $t$-level ideal of $R$. It is clear that $\mu$ is a fuzzy ideal of $R$ if and only if each $\mu^{t}, 0 \leq t \leq \mu(0)$, is an ideal of $R$. Without loss of generality, throughout this paper, we tacitly assume that $\mu(0)=1$ for every fuzzy ideal $\mu$ of $R$.

Let $a \in R, 0<\lambda \leq 1$. A fuzzy point $a^{\lambda}$ of $R$ is a fuzzy subset $a^{\lambda}: R \rightarrow[0,1]$ defined by for all $r \in R, a^{\lambda}(r)=\lambda$ if $r=a$ and 0 if $r \neq a$. The collection of all fuzzy points of $R$ is denoted by $\mathfrak{f P}(R)$. By $a^{\lambda} \in \mu$, we mean $\mu(a) \geq \lambda$, and $a^{\lambda} \in_{s} \mu$ means $\mu(a)>\lambda$. The collection of fuzzy points of $\mu$ is denoted by $\mathfrak{f P}(\mu)$. The point $a$ is called the support of $a^{\lambda}$ and $\lambda$ is its height. More generally, for a fuzzy subset $\mu$ of $R$, the support of $\mu$ is the set supp $\mu=\{x \in R: \mu(x)>0\}$. If $\mu$ is a fuzzy ideal then supp $\mu$ is an ideal of $R$.

The operations of sums and products on fuzzy subsets $\mu$ and $\nu$ of $R$ are defined as follows:

$$
\begin{aligned}
& (\mu+v)(x)=\sup \left\{\mu\left(x_{1}\right) \wedge v\left(x_{2}\right): x=x_{1}+x_{2}, x_{1}, x_{2} \in R\right\}, \\
& (\mu \circ v)(x)=\sup \{\mu(r) \wedge v(s): x=r s, r, s \in R\}, \\
& \quad(\mu \nu)(x)=\sup \left\{\bigwedge_{i=1}^{n}\left(\mu\left(r_{i}\right) \wedge v\left(s_{i}\right)\right): x=\sum_{i=1}^{n} r_{i} s_{i}, r_{i}, s_{i} \in R, n \in \mathbb{N}\right\} .
\end{aligned}
$$

If $x$ has no such decomposition, the sum and the product take the value 0 at $x$. 
For later use, we state without proof that the product distributes over addition for fuzzy ideals, viz. $\mu(\nu+\omega)=\mu \nu+\mu \omega$, see [9, Proposition 2.2.8]. Also, $\mu^{k} \mu^{r}=\mu^{k+r}$ and $\left(\mu^{k}\right)^{r}=\mu^{k r}$ for all $k, r \in \mathbb{N}$. If $a_{1}^{\lambda_{1}}, \ldots, a_{n}^{\lambda_{n}}$ are $n$ fuzzy points of $R$, then the fuzzy ideal $\mu$, generated by $a_{1}^{\lambda_{1}}, \ldots, a_{n}^{\lambda_{n}}$, is defined as $\sum_{i=1}^{n} x_{R} a_{i}^{\lambda_{i}}$ using the above definitions of sums and products. Such a $\mu$ is denoted by $\left\langle a_{1}^{\lambda}, a_{2}^{\lambda_{2}}, \ldots, a_{n}^{\lambda_{n}}\right\rangle$ and is called a finitely generated fuzzy ideal. The following two propositions deal with the product of fuzzy ideals generated by fuzzy points the proofs of which are found in [11].

Proposition 2.1. The fuzzy ideal $\left\langle a_{1}^{\lambda_{1}} a_{2}^{\lambda_{2}}\right\rangle=\left\langle a_{1}^{\lambda_{1}}\right\rangle\left\langle a_{2}^{\lambda_{2}}\right\rangle$ for $a_{1}^{\lambda_{1}}, a_{2}^{\lambda_{2}} \in$ $\mathfrak{f P}(R)$.

A consequence of Proposition 2.1 is the following proposition.

Proposition 2.2. If $v=\left\langle a_{1}^{\lambda_{1}}, a_{2}^{\lambda_{2}}\right\rangle$, then $v^{2}=\left\langle\left(a_{1}^{2}\right)^{\lambda_{1}}, a_{1}^{\lambda_{1}} a_{2}^{\lambda_{2}},\left(a_{2}^{2}\right)^{\lambda_{2}}\right\rangle$, where $v^{2}$ means the product $v v$.

Generally, if $v=\left\langle a_{1}^{\lambda_{1}}, a_{2}^{\lambda_{2}}, \ldots, a_{k}^{\lambda_{k}}\right\rangle$ for some $k \in \mathbb{N}$, then $v^{n}=\left\langle\left\{\left(a_{1}^{r_{1}}\right)^{\lambda_{1}} \ldots\right.\right.$ $\left.\left.\left(a_{k}^{r_{k}}\right)^{\lambda_{k}}: r_{1}+r_{2}+\cdots+r_{k}=n\right\}\right\rangle$, where we assume that $\lambda_{i}=1$ when $r_{i}=0$.

NoTE 2.3. We observe that the support supp $\mu$ of a finitely generated fuzzy ideal $\mu$ (finitely generated fuzzy submodule $\mu$ ) is finitely generated. Further, $\mu$ is finite-valued. Conversely, we have the following proposition.

Proposition 2.4. If $R$ is a Noetherian ring, then every finite-valued fuzzy ideal is finitely generated.

Proof. See [6, Proposition 3.9].

If $\omega$ is a fuzzy ideal of $R$, there are two equivalent ways in which we can define the radical. Given a fuzzy ideal $\omega$, the nil radical of $R \omega$ is defined by $R \omega(x)=\bigvee\left\{\omega\left(x^{n}\right): n \in \mathbb{N}\right\}, x \in R$, and the prime radical of $\omega$ is defined as $\bigwedge\{v: \omega \leq v, v$ is a prime fuzzy ideal of $R\}$. That they are indeed equivalent is shown in [11]. We denote either of the radical of $\omega$ by $\sqrt{\omega}$. The radical $\sqrt{\omega}$ is a fuzzy ideal of $R$ such that $\omega \leq \sqrt{\omega}$. A fuzzy ideal $\omega$ in $R$ is a maximal fuzzy ideal if $\omega \leq v, v$ a fuzzy ideal of $R$, implies $v=R$ or $\omega^{1}=v^{1}$ [14]. A fuzzy ideal $\omega$ is a maximal fuzzy ideal of $R$ if and only if $\operatorname{Im}(\omega)=\{1, t\}$ for some $t \in[0,1)$ and $\omega^{1}$ is a maximal ideal of $R$. The fuzzy Jacobson radical of $R$ is the infimum (intersection) of all maximal fuzzy ideals of $R$.

In the paragraph preceding Proposition 2.1, we defined the product of two fuzzy subsets $\mu$ and $v$. If one of them is a fuzzy point and the other is a fuzzy ideal, the product can be viewed as follows: let $\mu$ be a fuzzy ideal of $R$ and $r^{\lambda} \in \mathfrak{f} \mathfrak{Q}(R)$. The product $r^{\lambda} \mu$ is defined by $r^{\lambda} \mu(x)=\bigvee\left\{\lambda \wedge \bigwedge_{i=1}^{n} \mu\left(y_{i}\right): x=\right.$ $\left.\sum_{i=1}^{n} r y_{i}\right\}$, for $x \in R$. If $x$ has no such decomposition, then $r^{\lambda} \mu(x)=0$. We note that $r^{\lambda} \mu=\mu r^{\lambda}$. The residual fuzzy ideal $\mu: R$ of $R$ is characterised by $r^{\lambda} \in \mu: R$ if and only if $r^{\lambda} R \leq \mu$. More generally, the residual fuzzy ideal $\mu: \nu$ is characterised by, firstly, $\mu \leq v$ and, secondly, $r^{\lambda} \in \mu: v$ if and only if $r^{\lambda} v \leq \mu$. 
In terms of fuzzy points $\mu: R=\bigvee\left\{r^{\lambda} \in \mathfrak{f} \mathfrak{P}(R): r^{\lambda} R \leq \mu\right\}$. In this paper, we only have occasion to use the residual $\mu:\left\langle a^{\lambda}\right\rangle$ where $\left\langle a^{\lambda}\right\rangle$ is the fuzzy ideal generated by $a^{\lambda}$.

3. Primary decomposition. In this section, we describe a method of decomposing a fuzzy ideal as an intersection of primary fuzzy ideals. Assuming the existence of such a decomposition, we prove some uniqueness results. Most of the results of this section are well known in the literature [11, 12], albeit proved using a different technique from ours. We first recall the definitions of prime and primary fuzzy ideals.

A fuzzy ideal $\mu$ is called a prime (primary) fuzzy ideal in $R$ if $\mu \neq R$ and, for $r^{\beta}, s^{\lambda} \in \mathfrak{f f O}(R), r^{\beta} s^{\lambda} \in \mu$ implies either $r^{\beta} \in \mu$ or $s^{\lambda} \in \mu\left(s^{\lambda} \in \sqrt{\mu}\right)$, and is called $\nu$-primary if, firstly, $\mu$ is primary and, secondly, $v=\sqrt{\mu}$.

DEFINITION 3.1. Let $\left\{v_{i}: i=1,2, \ldots, n\right\}$ be a collection of prime fuzzy ideals and $\left\{\mu_{i}: i=1,2, \ldots, n\right\}$ a finite collection of $v_{i}$-primary fuzzy ideals of $R$. Then, $\mu=\bigwedge_{i=1}^{n} \mu_{i}$ is called a primary decomposition of $\mu$.

Note 3.2. This decomposition is said to be reduced or irredundant, (we use the two terms interchangeably) if (1) the $v_{1}, v_{2}, \ldots, v_{n}$ are all distinct and (2) $\mu_{j} \nsupseteq \bigwedge_{i=1, i \neq j}^{n} \mu_{i}$, for all $j=1,2, \ldots, n$.

Proposition 3.3. Let $\mu$ be a $v$-primary fuzzy ideal of $R$ and $r^{\lambda} \in \mathfrak{f P}(R)$. Then, the following are satisfied:

(i) if $r^{\lambda} \in \mu$, then $\mu:\left\langle r^{\lambda}\right\rangle=R$;

(ii) if $r^{\lambda} \notin \mu$, then $\mu:\left\langle r^{\lambda}\right\rangle$ is a $v$-primary fuzzy ideal of $R$. This implies, in particular, $v=\sqrt{\mu:\left\langle r^{\lambda}\right\rangle}$.

Proof. (i) Let $r^{\lambda} \in \mu$. For convenience, throughout the proof, $\omega$ denotes the fuzzy ideal generated by $r^{\lambda}$, that is, $\omega=\left\langle r^{\lambda}\right\rangle$. Then, for $r \in R, r^{1} \omega \leq \mu$. That is, $r^{1} \in \mu: \omega$ for all $r \in R$. Therefore, $\mu:\left\langle r^{\lambda}\right\rangle=R$.

(ii) Let $r^{\lambda} \notin \mu$. Consider $r_{1}^{\lambda_{1}} r_{2}^{\lambda_{2}} \in \mu$ : $\omega$. That is, $r_{1}^{\lambda_{1}} r_{2}^{\lambda_{2}} \omega \leq \mu$. Since $\mu$ is primary, either $r_{1}^{\lambda_{1}} \in \sqrt{\mu}$ or $r_{2}^{\lambda_{2}} \in \sqrt{\mu}$.

Let $\epsilon>0$. Then, there exist positive integers $n_{1}$ and $n_{2}$ such that either $\left(r_{1}^{n_{1}}\right)^{\lambda_{1}-\epsilon} \in \mu$ or $\left(r_{2}^{n_{2}}\right)^{\lambda_{2}-\epsilon} \in \mu$. Therefore, $\left(r_{1}^{n_{1}}\right)^{\lambda_{1}-\epsilon} \omega \leq \mu$ or $\left(r_{2}^{n_{2}}\right)^{\lambda_{2}-\epsilon} \omega \leq \mu$. This implies $r_{1}^{\lambda_{1}-\epsilon} \in \sqrt{\mu: \omega}$ or $r_{2}^{\lambda_{2}-\epsilon} \in \sqrt{\mu: \omega}$. As $\epsilon>0$ is arbitrary, either $r_{1}^{\lambda_{1}} \in \sqrt{\mu: \omega}$ or $r_{2}^{\lambda_{2}} \in \sqrt{\mu: \omega}$. This proves that $\mu:\left\langle r^{\lambda}\right\rangle$ is primary.

We now claim that $\sqrt{\mu:\left\langle r^{\lambda}\right\rangle}=\sqrt{\mu}=v$.

Let $s^{\beta} \in \sqrt{\mu}$. For $\epsilon>0$, there exists a positive integer $n$ such that $\left(s^{n}\right)^{\beta-\epsilon} \in \mu$. Since $\omega \leq R,\left(s^{n}\right)^{\beta-\epsilon} \in \mu: \omega$. As $\epsilon$ is arbitrary, $s^{\beta} \in \sqrt{\mu: \omega}$. Therefore, $\sqrt{\mu} \leq$ $\sqrt{\mu:\left\langle r^{\lambda}\right\rangle}$. The reverse inclusion follows from a similar argument. Therefore, $\nu=\sqrt{\mu:\left\langle r^{\lambda}\right\rangle}$.

Proposition 3.4. Let $\omega$ be a prime fuzzy ideal and $\omega_{1}, \omega_{2}, \ldots, \omega_{n}$ any $n$ fuzzy ideals of $R$. Then, the following statements are equivalent: 
(i) $\omega$ contains $\omega_{j}$ for some $j$,

(ii) $\omega \geq \bigwedge_{i=1}^{n} \omega_{i}$,

(iii) $\omega \geq \prod_{i=1}^{n} \omega_{i}$.

Proof. The implications (i) $\Rightarrow$ (ii) $\Rightarrow$ (iii) are straightforward. To show that (iii) $\Rightarrow$ (i), assume that $\omega \geq \prod_{i=1}^{n} \omega_{i}$. Suppose that $\omega$ does not contain $\omega_{j}$ for all $j=1,2, \ldots, n$. Then, for each $j$, there exists $r_{j}^{\lambda_{j}} \in \omega_{j} \backslash \omega$. Therefore, $r_{1}^{\lambda_{1}} r_{2}^{\lambda_{2}} \ldots$ $r_{n}^{\lambda_{n}} \notin \omega$ since $\omega$ is prime. But $r_{1}^{\lambda_{1}} r_{2}^{\lambda_{2}} \cdots r_{n}^{\lambda_{n}} \in \prod_{j=1}^{n} \omega_{j}$. This is a contradiction to our assumption. This completes the proof.

THEOREM 3.5. Let $\mu, \mu_{1}, \mu_{2}, \ldots, \mu_{n}$ be a set of $n+1$ fuzzy ideals of $R$ such that $\mu=\mu_{1} \wedge \mu_{2} \wedge \cdots \wedge \mu_{n}$ is a reduced primary decomposition of $\mu$. Let $\omega$ be a prime fuzzy ideal of $R$. Then, the following statements are equivalent:

(i) $\omega=v_{i}$ for some $i$ where $v_{i}=\sqrt{\mu_{i}}$,

(ii) there exists a fuzzy point $r^{\lambda} \in R$ such that $\mu:\left\langle r^{\lambda}\right\rangle$ is a $\omega$-primary fuzzy ideal,

(iii) there exists a fuzzy point $r^{\lambda} \in R$ such that $\omega=\sqrt{\mu:\left\langle r^{\lambda}\right\rangle}$.

Proof. (i) $\Rightarrow$ (ii). There exists a fuzzy point $r^{\lambda} \in \bigwedge_{j=1}^{n} \mu_{j} \backslash \mu_{i}$ since the decomposition is reduced. It is easily shown that $\left(\bigwedge_{j=1}^{n} \mu_{j}\right):\left\langle r^{\lambda}\right\rangle=\bigwedge\left(\mu_{j}:\left\langle r^{\lambda}\right\rangle\right)$. By Proposition 3.3, $\mu_{i}:\left\langle r^{\lambda}\right\rangle$ is a $v_{i}$-primary fuzzy ideal and $\mu_{j}:\left\langle r^{\lambda}\right\rangle=R$ for $j \neq i$. Hence, $\mu:\left\langle r^{\lambda}\right\rangle=\bigwedge_{j=1}^{n}\left(\mu_{j}:\left\langle r^{\lambda}\right\rangle\right)=\mu_{i}:\left\langle r^{\lambda}\right\rangle$ since others are $R$. Therefore, $\mu:\left\langle r^{\lambda}\right\rangle$ is $\omega$-primary because $\omega=v_{i}$.

(ii) $\Rightarrow$ (iii) is clear.

(iii) $\Rightarrow$ (i). Let $r^{\lambda} \in R$ such that $\omega=\sqrt{\mu:\left\langle r^{\lambda}\right\rangle}$. Then, $\omega=\bigwedge_{i=1}^{n}\left\{\sqrt{\mu_{i}:\left\langle r^{\lambda}\right\rangle}\right.$ such that $\left.r^{\lambda} \in R\right\}$ since if $r^{\lambda} \in \mu_{i}$ then $\mu_{i}:\left\langle r^{\lambda}\right\rangle=R$ or if $r^{\lambda} \notin \mu_{i}$ then $\omega=\bigwedge_{i=1}^{n} v_{i}$ by Proposition 3.3. Now, by Proposition 3.4, $\omega \geq v_{j}$ for some $j$ since $\omega=\bigwedge_{j=1}^{n} v_{j}$. Therefore, $\omega=v_{j}$ for some $j$.

THEOREM 3.6 (the first uniqueness theorem). Suppose that a fuzzy ideal $\mu$ has a reduced primary decomposition in the form $\mu=\mu_{1} \wedge \mu_{2} \wedge \cdots \wedge \mu_{n}$ with $v_{i}=\sqrt{\mu_{i}}$ for $i=1, \ldots, n$. Also, suppose that $\mu=\eta_{1} \wedge \eta_{2} \wedge \cdots \wedge \eta_{m}$ with $\xi_{j}=\sqrt{\eta_{j}}$ for $j=1, \ldots, m$ is another reduced primary decomposition of $\mu$. Then, $n=m$ and $\left\{v_{1}, v_{2}, \ldots, v_{n}\right\}=\left\{\xi_{1}, \xi_{2}, \ldots, \xi_{m}\right\}$.

Proof. Firstly, we observe that $\xi_{1}$ is a prime fuzzy ideal of $R$. Set $\xi_{1}=\omega$. Consider a fuzzy point $r^{\lambda} \in\left(\bigwedge_{j=2}^{m} \eta_{j}\right) \backslash \eta_{1}$. By Proposition 3.3, $\eta_{1}:\left\langle r^{\lambda}\right\rangle$ is a $\xi_{1}$-primary fuzzy ideal of $R$ and $\xi_{1}=\sqrt{\eta_{1}:\left\langle r^{\lambda}\right\rangle}$. Since $r^{\lambda} \in \bigwedge_{j=2}^{m} \eta_{j}$, we have $\eta_{j}:\left\langle r^{\lambda}\right\rangle=R$, for all $j=2,3, \ldots, m$. Therefore, $\mu:\left\langle r^{\lambda}\right\rangle=\eta_{1}:\left\langle r^{\lambda}\right\rangle$ is a $\omega$-primary ideal of $R$. Thus, we have a fuzzy point $r^{\lambda} \in R$ such that $\omega=\sqrt{\eta_{1}:\left\langle r^{\lambda}\right\rangle}=$ $\sqrt{\mu:\left\langle r^{\lambda}\right\rangle}$.

Secondly, by Theorem 3.5, there exists an $i \in\{1,2, \ldots, n\}$ such that $\omega=v_{i}$. That is, $\xi_{1}=v_{i}$ for some $1 \leq i \leq n$. Repeating the process, we get $\xi_{j}=v_{k}$ for some $1 \leq k \leq n$ for each $j=2, \ldots, m$. This implies that $\left\{\xi_{1}, \xi_{2}, \ldots, \xi_{m}\right\} \subseteq$ $\left\{v_{1}, v_{2}, \ldots, v_{n}\right\}$. Therefore, $m \leq n$. Reversing the roles of $\xi_{j}$ 's and $v_{i}$ 's, we get 
the reverse inclusion of the above subsets, implying $n \leq m$. This completes the proof.

DEFINITION 3.7. The set $\left\{v_{1}, v_{2}, \ldots, v_{n}\right\}$, as stated in the above theorem, is known as the set of associated prime ideals of $\mu$ and is denoted by $\operatorname{Ass}_{R}(\mu)$. In this case, we say that each $v_{i}$ belongs to $\mu$.

For the next proposition, we require the notion of minimal prime fuzzy ideal. We recall that a prime fuzzy ideal $\mu$ containing $v$ is said to be minimal if $v \leq \mu$ and, whenever a prime $\omega$ satisfies the inequality $v \leq \omega \leq \mu$, then $\omega=v$ or $\omega=\mu$.

Proposition 3.8. Let $\mu$ be a fuzzy ideal with a primary decomposition, and let $v$ be a prime fuzzy ideal such that $v \geq \mu$. Then, $v$ is a minimal prime fuzzy ideal containing $\mu$ if and only if $v$ is a minimal member of $\operatorname{Ass}_{R}(\mu)$.

Proof. Let $\mu=\bigwedge_{i=1}^{n} \mu_{i}$ be a reduced primary decomposition of $\mu$. Let $\nu_{i}=$ $\sqrt{\mu_{i}}$ for $i=1,2, \ldots, n$ be the associated prime ideals of $\mu$. We note that $v \geq \mu$ if and only if $\nu=\sqrt{v} \geq \sqrt{\mu}$. Now, $\sqrt{\mu}=\bigwedge_{i=1}^{n} \sqrt{\mu_{i}}=\bigwedge_{i=1}^{n} v_{i}$. So, $v \geq \mu$ if and only if $v \geq \bigwedge_{i=1}^{n} v_{i}$. Therefore, $v \geq v_{j}$ for some $j$ between 1 and $n$ by Proposition 3.4.

$(\Rightarrow)$. Firstly, we observe that $v_{j} \geq \mu$ because $v_{j}=\sqrt{\mu_{j}} \geq \mu_{j} \geq \mu$. This is true for all $j=1,2, \ldots, n$. From above, $v \geq v_{j}$ for some $j$ between 1 and $n$. Now, minimality of $v$ implies $v=v_{j}$. Therefore, $v$ is a minimal member of $\operatorname{Ass}_{R}(\mu)$.

$(\Leftarrow) . v \geq \mu$ implies that there exists a minimal prime fuzzy ideal $\omega$ such that $v \geq \omega \geq \mu$. Therefore, there exists a $v_{j} \in \operatorname{Ass}_{R}(\mu)$ such that $v \geq \omega \geq v_{j} \geq \mu$ as stated above. Minimality of $v$ in $\operatorname{Ass}_{R}(\mu)$ implies that $v=\omega=v_{j}$ which, in turn, implies that $\nu$ is a minimal prime fuzzy ideal containing $\mu$.

Note 3.9. Minimal members of $\operatorname{Ass}_{R}(\mu)$ are called the minimal or isolated primes of $\mu$. The other members of $\operatorname{Ass}_{R}(\mu)$ are called embedded primes of $\mu$.

LEMMA 3.10. Let $\omega$ be a $v$-primary fuzzy ideal of $R$. If $a^{\lambda} \notin \nu$, then $\omega$ : $\left\langle a^{\lambda}\right\rangle=\omega$.

Proof. Clearly, $\omega:\left\langle a^{\lambda}\right\rangle \geq \omega$. Let $r^{\beta} \in \omega:\left\langle a^{\lambda}\right\rangle$. Then, $r^{\beta} a^{\lambda} \in \omega$. But, by hypothesis, $a^{\lambda} \notin v=\sqrt{\omega}$. Therefore, $r^{\beta} \in \omega$ since $\omega$ is $v$-primary. This completes the proof.

THEOrem 3.11 (the second uniqueness theorem). Let $\mu$ be a fuzzy ideal of $R$. Suppose that $\mu$ has the following two reduced primary decompositions:

$$
\begin{aligned}
& \mu=\mu_{1} \wedge \mu_{2} \wedge \cdots \wedge \mu_{n}, \quad \text { with } v_{i}=\sqrt{\mu_{i}} \text { for } i=1, \ldots, n, \\
& \mu=\xi_{1} \wedge \xi_{2} \wedge \cdots \wedge \xi_{n}, \quad \text { with } v_{i}=\sqrt{\xi_{i}} \text { for } i=1, \ldots, n .
\end{aligned}
$$

Then, for each $i$ for which $\nu_{i}$ is a minimal prime fuzzy ideal belonging to $\mu$, $\mu_{i}=\xi_{i}$. 
Proof. For $n=1, \operatorname{Ass}_{R}(\mu)=\left\{v_{1}\right\}$. So, $\mu_{1}=\xi_{1}$. For $n>1$, let $\mu_{i}$ be a minimal member of $\operatorname{Ass}_{R}(\mu)$. Then, there exists a fuzzy point $r^{\lambda} \in \bigwedge_{j=1, j \neq i}^{n} v_{j} \backslash v_{i}$. (Otherwise, $v_{i} \geq \bigwedge_{j=1, j \neq i}^{n} v_{j}$ which, in turn, will imply that $v_{i}>v_{j}$ for some $j$, contradicting the minimality of $v_{i}$.) Now, for $\epsilon>0, r^{\lambda-\epsilon} \notin v_{i}$ and $r^{\lambda-\epsilon} \in \bigwedge_{j=1, j \neq i}^{n} v_{j}$. So, we can assume without loss of generality that $r^{\lambda}$ is strictly in $\bigwedge_{j=1, j \neq i}^{n} \nu_{j} \backslash v_{i}$. Thus, there exists a positive integer $m_{j}$ such that $\left(r^{m_{j}}\right)^{\lambda} \in \mu_{j}$ for every $j \neq i$. Set $t \geq \max \left\{m_{j}: j=1,2, \ldots, n, j \neq i\right\}$. Then, $\left(r^{t}\right)^{\lambda} \in \mu_{j}$ and $\left(r^{t}\right)^{\lambda} \notin v_{i}$. Therefore, $\mu:\left\langle\left(r^{t}\right)^{\lambda}\right\rangle=\bigwedge\left(\mu_{j}\right):\left\langle\left(r^{t}\right)^{\lambda}\right\rangle=\mu_{i}:\left\langle\left(r^{t}\right)^{\lambda}\right\rangle$ since $\mu_{j}:\left\langle\left(r^{t}\right)^{\lambda}\right\rangle=R$ for $j \neq i$. Now, $\mu_{i}:\left\langle\left(r^{t}\right)^{\lambda}\right\rangle=\mu_{i}$ by Lemma 3.10 since $\mu_{i}$ is assumed to be $v_{i}$-primary. Similarly, $\xi_{i}=\mu_{i}:\left\langle\left(r^{t}\right)^{\lambda}\right\rangle$ for a large enough value for $t$. Thus, we have $\mu_{i}=\xi_{i}$.

4. Irreducible decompositions. Throughout this section and the next one, $R$ denotes a Noetherian commutative ring with unity.

Definition 4.1. Let $\mu$ be a fuzzy ideal of $R$. It is said to be irreducible if $\mu \neq R$ and whenever $\mu=\mu_{1} \wedge \mu_{2}$, where $\mu_{1}$ and $\mu_{2}$ are fuzzy ideals of $R$, then $\mu=\mu_{1}$ or $\mu=\mu_{2}$.

Proposition 4.2. Let $\mu$ be an irreducible fuzzy ideal of $R$. Then, $\operatorname{Im}(\mu)=$ $\{1, t\}$ for some $0 \leq t<1$, and the top cut $\mu^{1}$ is irreducible.

Proof. Suppose that $\mu(z)=t_{1}<t_{2}=\mu(y) \neq 1$ for some $z, y \in R$. Define

$$
\begin{aligned}
& \mu_{1}(x)= \begin{cases}1, & x \in \mu^{1}, \\
\mu(x), & x \in \mu^{t_{2}} \backslash \mu^{1}, \\
t_{2} & \text { otherwise }\end{cases} \\
& \mu_{2}(x)= \begin{cases}1, & x \in \mu^{t_{2}}, \\
\mu(x), & \text { otherwise }\end{cases}
\end{aligned}
$$

Firstly, we note that $\mu_{1}, \mu_{2} \geq \mu$ and $\mu_{1} \neq \mu, \mu_{2} \neq \mu$. Secondly,

$$
\begin{aligned}
\left(\mu_{1} \wedge \mu_{2}\right)(x) & = \begin{cases}1, & x \in \mu^{1}, \\
\mu(x), & \text { otherwise }\end{cases} \\
& =\mu(x) .
\end{aligned}
$$

This contradicts the irreducibility of $\mu$. Therefore, $|\operatorname{Im}(\mu)|=2$.

Further, suppose that $\mu^{1}$ is reducible. Then, there exist two proper ideals $J_{1}$ and $J_{2}$ of $R$ such that $\mu^{1}=J_{1} \cap J_{2}$. For $i=1,2$, let

$$
\mu_{i}(x)= \begin{cases}1, & x \in J_{i} \\ \mu(x) & \text { otherwise }\end{cases}
$$


We note that $\mu_{i} \geq \mu$ and $\mu_{i} \neq \mu$ for $i=1,2$,

$$
\begin{aligned}
\left(\mu_{1} \wedge \mu_{2}\right)(x) & = \begin{cases}1, & x \in \mu^{1}, \\
\mu(x) & \text { otherwise }\end{cases} \\
& =\mu(x) .
\end{aligned}
$$

This is a contradiction to the irreducibility of $\mu$. Therefore, $\mu^{1}$ is irreducible.

With each irreducible crisp ideal of $R$, there is an associated irreducible fuzzy ideal of $R$ as the following proposition shows.

Proposition 4.3. Let I be an irreducible ideal of $R$ and define a fuzzy ideal $\mu$ as $\mu(x)=1$ if $x \in I$ and $\alpha$ if $x \notin I$ for some $0 \leq \alpha<1$. Then $\mu$ is irreducible.

Proof. Suppose that there exist fuzzy ideals $\mu_{1}, \mu_{2} \neq \mu$ such that $\mu=$ $\mu_{1} \wedge \mu_{2}$. This implies the existence of an $x \in R$ such that $\mu_{1}(x)>\mu(x)$. Similarly, there is a $y \in R$ such that $\mu_{2}(y)>\mu(y)$. Consider the ideals generated by $I$ and $x,(\langle I, x\rangle)$, and $I$ and $y,(\langle I, y\rangle)$. Clearly, $I$ is contained in $\langle I, x\rangle \cap\langle I, y\rangle$, and also, $I$ is distinct from each of $\langle I, x\rangle$ and $\langle I, y\rangle$. Let $a \in\langle I, x\rangle \cap\langle I, y\rangle$, then $a=r_{1} x_{1}+s_{1} y=r_{2} x_{2}+s_{2} x$, where $x_{1}, x_{2} \in I$ and $r_{1}, r_{2}, s_{1}, s_{2} \in R$. Now, $\mu\left(r_{1} x_{1}-r_{2} x_{2}\right)=1=\mu\left(s_{2} x-s_{1} y\right)$. This implies that $\mu\left(r_{1} x_{1}\right)=\mu\left(r_{2} x_{2}\right)$ and $\mu\left(s_{2} x\right)=\mu\left(s_{1} y\right)$. Also, $\mu_{1}\left(s_{2} x\right) \geq \mu_{1}(x)>\mu(x) \geq \alpha$ and $\mu_{2}\left(s_{2} x\right)=\mu_{2}\left(s_{2} x-\right.$ $\left.s_{1} y+s_{1} y\right) \geq \mu_{2}\left(s_{2} x-s_{1} y\right) \wedge \mu_{2}\left(s_{1} y\right)=\mu_{2}\left(s_{1} y\right) \geq \mu_{2}(y)>\mu(y) \geq \alpha$. Therefore, $\left(\mu_{1} \wedge \mu_{2}\right)\left(s_{2} x\right)>\alpha$ which implies that $\mu\left(s_{2} x\right)>\alpha$. Hence, $\mu\left(s_{2} x\right)=1$. This implies that $a \in I$ and so $\langle I, y\rangle \cap\langle I, x\rangle \subseteq I$. Therefore, $I=\langle I, y\rangle \cap\langle I, x\rangle$. This contradicts the irreducibility of $I$.

COROLlaRY 4.4. If $\mu$ is a fuzzy ideal such that $\mu^{1}$ is irreducible and $\operatorname{Im}(\mu)=$ $\{1, \alpha\}$, then $\mu$ is irreducible.

Note 4.5. A prime fuzzy ideal is necessarily irreducible; however, the converse need not hold as the following example shows.

EXAMPLE 4.6. Let $R$ be the $\operatorname{ring} \mathbb{Z}+\mathbb{Z}$. Consider $I=4 \mathbb{Z}+\mathbb{Z}$. We claim that $I$ is irreducible. Suppose not. Then, there exist two ideals $J_{1}$ and $J_{2}$ such that $J_{i} \neq I ; I \subset J_{i}$ for $i=1,2$ where $I=J_{1} \cap J_{2}$. It is an easy exercise to check that $J_{1}$ and $J_{2}$ are of the form $k_{1} \mathbb{Z}+k_{2} \mathbb{Z}$ and $m_{1} \mathbb{Z}+m_{2} \mathbb{Z}$ for some positive integers $k_{1}, k_{2}, m_{1}$ and $m_{2}$, respectively. Then, $4 \mathbb{Z}+\mathbb{Z}=\left(k_{1} \mathbb{Z}+k_{2} \mathbb{Z}\right) \cap\left(m_{1} \mathbb{Z}+m_{2} \mathbb{Z}\right)=$ $\left(k_{1} \mathbb{Z} \cap m_{1} \mathbb{Z}\right)+\left(k_{2} \mathbb{Z} \cap m_{2} \mathbb{Z}\right)$, which implies trivially $k_{2}=1=m_{2}$. Further, $4 \mathbb{Z} \subset$ $k_{1} \mathbb{Z} \cap m_{1} \mathbb{Z}$. Therefore, $k_{1}, m_{1}$ divide 4 . Each possible value for $k_{1}$ and $m_{1}$ as a divisor of 4 leads to an absurd equality. Thus, the claim is proved. Now, we obtain the required example by defining $\mu(x)$ to be equal to 1 if $x \in 4 \mathbb{Z}+\mathbb{Z}$ and $1 / 3$ otherwise.

Proposition 4.7. Let $\mu$ be an irreducible fuzzy ideal of $R$. Then, $\mu$ is a primary fuzzy ideal. 
Proof. Since $\mu$ is irreducible, $\mu \neq R ; \operatorname{Im}(\mu)=\{1, \alpha\}$ for some $0 \leq \alpha<1$ and $\mu^{1}$ is irreducible. Now, we need only to show that $\mu^{1}$ is primary. To this end, consider $r s \in \mu^{1}$ and $s \notin \mu^{1}$. Since $R$ is Noetherian, we can find a positive integer $n$ such that the ascending chain $\mu^{1}:\langle r\rangle \subseteq \mu^{1}:\left\langle r^{2}\right\rangle \subseteq \mu^{1}:\left\langle r^{3}\right\rangle \subseteq \cdots$ ends with $\mu^{1}:\left\langle r^{n}\right\rangle=\mu^{1}:\left\langle r^{n+1}\right\rangle=\cdots$.

ClaIm. The top cut $\mu^{1}=\left(\mu^{1}+r^{n} R\right) \cap\left(\mu^{1}+R s\right)$.

Clearly, $\mu^{1} \subseteq\left(\mu^{1}+r^{n} R\right) \cap\left(\mu^{1}+R s\right)$. For the reverse inclusion, consider, $y \in$ $\left(\mu^{1}+r^{n} R\right) \cap\left(\mu^{1}+R s\right)$. Then, $y=s_{1}+r^{n} s_{2}=s_{3}+t s$ for $s_{1}, s_{3} \in \mu^{1}, s_{2} \in R$, and $t \in R$. The element $r y=r s_{1}+r^{n+1} s_{2}=r s_{3}+t(r s) \in \mu^{1}$, which implies that $r^{n+1} s_{2} \in \mu^{1}$, which, in turn, implies that $s_{2} \in \mu^{1}:\left\langle r^{n+1}\right\rangle$. Therefore, $s_{2} \in \mu^{1}$ : $\left\langle r^{n}\right\rangle$, which implies that $r^{n} s_{2} \in \mu^{1}$. Therefore, $y \in \mu^{1}$. This proves the claim.

Since $\mu^{1}$ is irreducible, either $\mu^{1}=\mu^{1}+r^{n} R$ or $\mu^{1}=\mu^{1}+R s$. But $s \in\left(\mu^{1}+\right.$ $R s) \backslash \mu^{1}$. Hence, $\mu^{1}=\mu^{1}+r^{n} R$, implying that $r^{n} R \subseteq \mu^{1}$. This completes the proof.

LEMmA 4.8. Let $\mu$ be a fuzzy ideal of $R$ such that $|\operatorname{Im}(\mu)|=2$. Then, $\mu$ can be expressed as a finite intersection of irreducible fuzzy ideals of $R$.

PROOF. The top cut $\mu^{1}$ is an intersection of a collection of irreducible ideals $J_{i}, i=1,2, \ldots, n$ of $R$ [13]. Suppose that $\operatorname{Im}(\mu)=\{1, t\}, 0 \leq t<1$. Define $v_{i}(x)=$ 1 if $x \in J_{i}$ and $t$ otherwise. Each $v_{i}$ is irreducible and $\mu=\bigwedge_{i=1}^{n} v_{i}$.

THEOREM 4.9. Let $\mu$ be a proper finite-valued fuzzy ideal of $R$. Then, $\mu$ can be expressed as a finite intersection of irreducible fuzzy ideals of $R$.

Proof. Firstly, we observe that $|\operatorname{Im}(\mu)|<\infty$, so we can assume that $\operatorname{Im}(\mu)=$ $\left\{1, t_{n}, t_{n-1}, \ldots, t_{1}\right\}$, where $1>t_{n}>t_{n-1}>\cdots>t_{1}$.

Let $\mu^{1}=\cap_{i=1}^{m_{1}} J_{1 i}, \mu^{t_{n}}=\cap_{i=1}^{m_{2}} J_{t_{n} i}, \ldots, \mu^{t_{2}}=\cap_{i=1}^{m_{n}} J_{t_{2} i}$ where $J_{11}, \ldots, J_{t_{n}}, \ldots$, $J_{t_{2} 1}, \ldots, J_{t_{2} m_{n}}$ are all irreducible ideals of $R$. Define the fuzzy ideals $v$ 's as follows:

$$
\begin{gathered}
v_{1 i}(x)= \begin{cases}1, & \text { if } x \in J_{1 i}, \\
t_{n}, & \text { otherwise, }\end{cases} \\
v_{t_{n} i}(x)= \begin{cases}1, & \text { if } x \in J_{t_{n} i}, \\
t_{n-1}, & \text { otherwise, }\end{cases} \\
\vdots \\
v_{t_{2} i}(x)= \begin{cases}1, & \text { if } x \in J_{t_{2} i}, \\
t_{1}, & \text { otherwise }\end{cases}
\end{gathered}
$$

Then, all the above $v_{1 i}, \ldots, v_{t_{2} i}, \ldots$ are irreducible. Further,

$$
\bigwedge_{i=1}^{m_{1}} v_{1 i}(x)= \begin{cases}1, & \text { if } x \in \cap_{i=1}^{m_{1}} J_{1 i}=\mu^{1} \\ t_{n}, & \text { otherwise }\end{cases}
$$




$$
\begin{gathered}
\bigwedge_{i=1}^{m_{2}} v_{t_{n} i}(x) \\
= \begin{cases}1, & \text { if } x \in \cap_{i=1}^{m_{2}} J_{t_{n} i}=\mu^{t_{n}}, \\
t_{n-1}, & \text { otherwise }\end{cases} \\
\vdots
\end{gathered}
$$

Therefore, $\mu=\left(\bigwedge v_{1 i}\right) \wedge\left(\bigwedge v_{t_{n} i}\right) \wedge \cdots \wedge\left(\bigwedge v_{t_{2} i}\right)$.

We end this section with the following corollary on the existence of primary decompositions. Theorem 4.9 tells us that every proper finite-valued fuzzy ideal of $R$ can be expressed as a finite intersection of irreducible fuzzy ideals of $R$. Now, from Proposition 4.7, we know that an irreducible fuzzy ideal is primary. Thus, we have the following corollary.

COROLLARY 4.10. Let $\mu$ be a proper finite-valued fuzzy ideal of $R$. Then, $\mu$ has a reduced primary decomposition.

5. Krull's intersection theorem. In general, it is not true that $\bigwedge_{n=1}^{\infty} \mu^{n}=0$ for a fuzzy ideal $\mu$ of $R$. But Krull proved that such equality holds under certain additional hypotheses in the crisp case [1]. In this section, we study his theorem in the fuzzy case.

LEMMA 5.1. Let $v$ be a fuzzy ideal of $R$. Suppose that $\sqrt{v}$ is a finitely generated fuzzy ideal of $R$. Then, there exists a natural numbern such that $(\sqrt{v})^{n} \leq v$.

Proof. Let $\sqrt{v}=\left\langle a_{1}^{\lambda_{1}}, a_{2}^{\lambda_{2}}, \ldots, a_{k}^{\lambda_{k}}\right\rangle$ for some positive integer $k$. Given an arbitrary $\epsilon>0$, there exists, for each $i$ between 1 and $k, n_{i} \in \mathbb{N}$ such that $\left(a_{i}^{n_{i}}\right)^{\lambda_{i}-\epsilon} \in v$. Set $n=1+\sum_{i=1}^{k}\left(n_{i}-1\right)$. By Proposition 2.2, $(\sqrt{v})^{n}=\left\langle\left\{\left(a_{1}^{r_{1}}\right)^{\lambda_{1}} \ldots\right.\right.$ $\left.\left.\left(a_{k}^{r_{k}}\right)^{\lambda_{k}}: r_{1}+r_{2}+\cdots+r_{k}=n\right\}\right\rangle$, where we assume that $\lambda_{i}=1$ when $r_{i}=0$. It is easy to see that an $r_{j} \geq n_{j}$ for some $1 \leq j \leq n$. Therefore, $\left(a_{j}^{r_{j}}\right)^{\lambda_{j}-\epsilon} \in \nu$. As $\epsilon$ is arbitrary, $(\sqrt{v})^{n} \leq v$.

Proposition 5.2. Let $\mu$ be a finite-valued proper fuzzy ideal of $R$ and $v a$ prime fuzzy ideal of $R$. Then, $v \in \operatorname{Ass}_{R}(\mu)$ if and only if there exists $a^{\lambda} \in \mathfrak{f} \vartheta(R)$ such that $\mu:\left\langle a^{\lambda}\right\rangle=v$.

Proof. $(\Leftarrow)$. Suppose that there exists a $a^{\lambda} \in \mathfrak{f} \mathcal{P}(R)$ such that $\mu:\left\langle a^{\lambda}\right\rangle=v$. Then, $\mu:\left\langle a^{\lambda}\right\rangle$ is prime, therefore $v$-primary. Then, by Theorem 3.5, $v=v_{i}$ for some $1 \leq i \leq n$ such that $\nu_{i}=\sqrt{\mu_{i}}$ where $\mu=\mu_{1} \wedge \mu_{2} \wedge \cdots \wedge \mu_{n}$ is a reduced primary decomposition, that is, $v \in \operatorname{Ass}_{R}(\mu)$.

$(\Rightarrow)$. Suppose that $v \in \operatorname{Ass}_{R}(\mu)$ where $\mu=\mu_{1} \wedge \mu_{2} \wedge \cdots \wedge \mu_{n}, v_{i}=\sqrt{\mu_{i}}$, is a reduced primary decomposition. Consider $\omega_{j}=\bigwedge_{i=1, i \neq j}^{n} \mu_{i}$. This implies that $\mu \leq \omega_{j} \npreceq \mu_{j}$ since $\mu=\bigwedge_{i=1}^{n} \mu_{i}$ is a reduced primary decomposition. Now, by Proposition 2.4, $v_{j}$ is finitely generated. So, Lemma 5.1 implies that there exists a positive integer $t$ such that $v_{j}^{t}=\left(\sqrt{\mu_{j}}\right)^{t} \leq \mu_{j}$. Now, $v_{j}^{t} \omega_{j} \leq \mu_{j} \omega_{j} \leq \mu_{j} \wedge \omega_{j}=$ $\mu$. Let $u$ be the least positive integer such that $v_{j}^{u} \omega_{j} \leq \mu$ but $v_{j}^{u-1} \omega_{j} \nless \mu$. 
Choose $a^{\lambda} \in v_{j}^{u-1} \omega_{j} \backslash \mu$. Then, $a^{\lambda} \in \omega_{j} \backslash \mu$. Now, $\mu_{i}:\left\langle a^{\lambda}\right\rangle=R$ for all $i=$ $1,2, \ldots, n, i \neq j$ and $\mu_{j}:\left\langle a^{\lambda}\right\rangle$ is $v_{j}$-primary by Proposition 3.3. Thus, $\mu:\left\langle a^{\lambda}\right\rangle=$ $\bigwedge_{i=1}^{n}\left(\mu_{i}:\left\langle a^{\lambda}\right\rangle\right)=\mu_{j}:\left\langle a^{\lambda}\right\rangle$. Therefore, $\mu:\left\langle a^{\lambda}\right\rangle$ is $v_{j}$-primary. Now, $\left\langle a^{\lambda}\right\rangle v_{j} \leq$ $v_{j}^{u} \omega_{j} \leq \mu$. Hence, $v_{j} \leq \mu:\left\langle a^{\lambda}\right\rangle \leq v_{j}$ since $\mu:\left\langle a^{\lambda}\right\rangle=\mu_{j}:\left\langle a^{\lambda}\right\rangle \leq \sqrt{\mu_{j}:\left\langle a^{\lambda}\right\rangle}=v_{j}$. This completes the proof.

THEOREM 5.3. Let $\mu$ be a finite-valued fuzzy ideal of $R$, and set $v=\bigwedge_{n=1}^{\infty} \mu^{n}$. Then $v=\mu v$.

Proof. The case when $\mu=R$ is clear. Now, suppose that $\mu \neq R$. Then, $\mu \nu \neq$ $R$ since $\mu \nu \leq \mu$. Thus, $\mu \nu$ has a primary decomposition by Corollary 4.10. Let $\mu \nu=\mu_{1} \wedge \mu_{2} \wedge \cdots \wedge \mu_{n}$ with $\nu_{i}=\sqrt{\mu_{i}}, i=1,2, \ldots, n$ be a reduced primary decomposition of $\mu \nu$. We need only to show that $v \leq \mu \nu$ since the reverse inequality is always true. Suppose that there is a natural number $i, 1 \leq i \leq n$, such that $\nu \npreceq \mu_{i}$. Then, there exists a fuzzy point $a^{\lambda} \in \nu \backslash \mu_{i}$. Now, $a^{\lambda} \mu \leq \mu \nu=$ $\bigwedge_{i=1}^{n} \mu_{i} \leq \mu_{i}$ and $\mu_{i}$ is $\nu_{i}$-primary. Therefore, $\mu \leq \sqrt{\mu_{i}}=\nu_{i}$ since $a^{\lambda} \notin \mu_{i}$, and $v_{i}$ is finitely generated by Proposition 2.4. Now, by Lemma 5.1, there exists a positive integer $t$ such that $v_{i}^{t} \leq \mu_{i}$, thus $v=\bigwedge_{n=1}^{\infty} \mu^{n} \leq \mu^{t} \leq v_{i}^{t} \leq \mu_{i}$. This contradicts our assumption that $v \nless \mu_{i}$. Therefore, $v \leq \mu_{i}$ for all $i=1,2, \ldots, n$, and hence, $v \leq \mu \nu$. This completes the proof.

LEMMA 5.4 (Nakayama's lemma). Let $\mu$ be a finitely generated fuzzy submodule of a module $M$ over a commutative ring $R$. Let $v$ be a fuzzy ideal of $R$ contained in the fuzzy Jacobson radical of $R$. If $\mu=\nu \mu$, then $\mu$ is the trivial fuzzy submodule.

NoTE 5.5. By the trivial fuzzy submodule of $M$, we mean, for all $x \in M$, $\mu(x)=1$ if $x=0$ and 0 if $x \neq 0$.

Proof. Since $\mu=v \mu$, supp $\mu=(\operatorname{supp} v)(\operatorname{supp} \mu)$. Set $I=\operatorname{supp} v$ be the crisp ideal in $R$. Let $2 \mathfrak{W}$ be any maximal ideal of $R$. Construct $\omega$ on $R$ as follows: $\omega(x)=1$ if $x \in \mathfrak{W}$ and 0 otherwise. Note that $\omega$ is a maximal fuzzy ideal of $R$. Now, $v$ is contained in the fuzzy Jacobson radical of $R$ which is itself contained in $\omega$. Therefore, $v \leq \omega$, implying $I=\operatorname{supp} v \subseteq \operatorname{supp} \omega=2$ W. Thus, $I$ is contained in the crisp Jacobson radical of $R$. By Nakayama's lemma for the crisp case, $\operatorname{supp} \mu=0$. This completes the proof.

THEOREM 5.6 (Krull's intersection theorem). Let $\mu$ be a finitely generated fuzzy ideal of $R$ such that $\mu$ is contained in the fuzzy Jacobson radical of $R$. Then $\bigwedge_{n=1}^{\infty} \mu^{n}=0$.

Proof. Let $v=\bigwedge_{n=1}^{\infty} \mu^{n}$. Since $\mu$ is finitely generated, $\mu$ is finite-valued, which, in turn, implies that $v$ is finite-valued. By Theorem 5.3, $v=\mu \nu$. Now, Nakayama's lemma implies that $v=0$.

ACKNOWLEDGMENT. This research was supported by the Foundation for Research and Development (FRD) of South Africa. 


\section{REFERENCES}

[1] T. W. Hungerford, Algebra, Graduate Texts in Mathematics, vol. 73, SpringerVerlag, New York, 1980.

[2] R. Kumar, Fuzzy irreducible ideals in rings, Fuzzy Sets and Systems 42 (1991), no. 3, 369-379.

[3] P. Lubczonok, Fuzzy vector spaces, Fuzzy Sets and Systems 38 (1990), no. 3, 329-343.

[4] B. B. Makamba and V. Murali, On prime fuzzy submodules and radicals, J. Fuzzy Math. 8 (2000), no. 4, 831-843.

[5] _ Primary decompositions of fuzzy submodules, J. Fuzzy Math. 8 (2000), no. 4, 817-829.

[6] _ On an algebra of fuzzy points on modules, J. Fuzzy Math. 9 (2001), no. 4, 799-806.

[7] D. S. Malik and J. N. Mordeson, Fuzzy prime ideals of a ring, Fuzzy Sets and Systems 37 (1990), no. 1, 93-98.

[8] _ _ Fuzzy primary representations of fuzzy ideals, Inform. Sci. 55 (1991), no. 1-3, 151-165.

[9] _ Radicals of fuzzy ideals, Inform. Sci. 65 (1992), no. 3, 239-252.

[10] _ R-primary L-representations of L-ideals, Inform. Sci. 88 (1996), no. 1-4, 227-246.

[11] J. N. Mordeson and D. S. Malik, Fuzzy Commutative Algebra, World Scientific Publishing, New Jersey, 1998.

[12] R. Sekaran, Fuzzy Ideals in Commutative Rings, Master's thesis, Rhodes University, South Africa, 1995.

[13] R. Y. Sharp, Steps in Commutative Algebra, London Mathematical Society Student Texts, vol. 19, Cambridge University Press, Cambridge, 1990.

[14] U. M. Swamy and K. L. N. Swamy, Fuzzy prime ideals of rings, J. Math. Anal. Appl. 134 (1988), no. 1, 94-103.

V. Murali: Department of Mathematics (Pure \& Applied), Rhodes University, Grahamstown 6140, South Africa

E-mail address: v.murali@ru.ac.za

B. B. Makamba: Department of Mathematics, University of Fort Hare, Alice 5700, South Africa

E-mail address: makamba@ufh.ac.za 


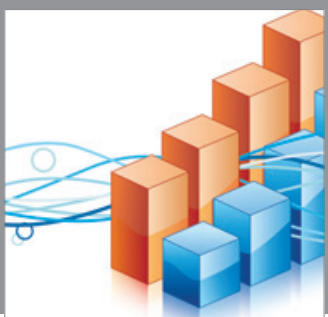

Advances in

Operations Research

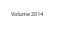

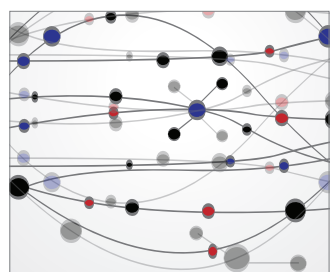

\section{The Scientific} World Journal
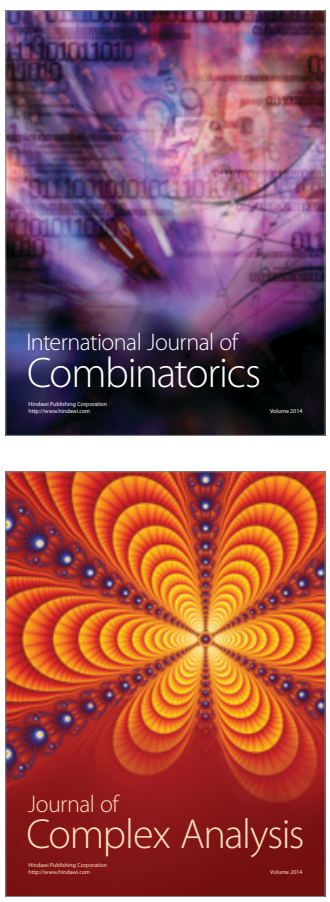

International Journal of

Mathematics and

Mathematical

Sciences
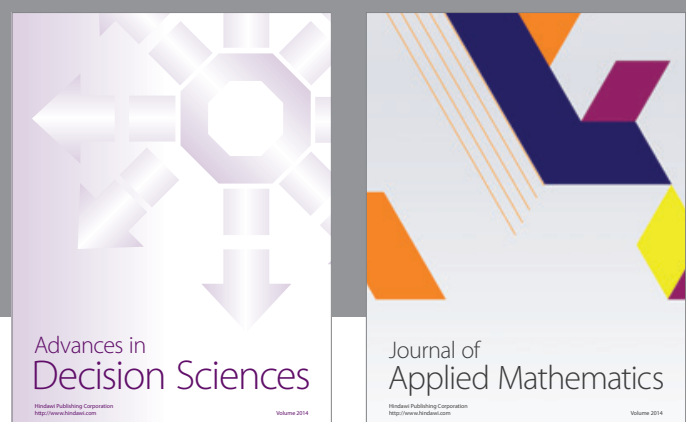

Journal of

Applied Mathematics
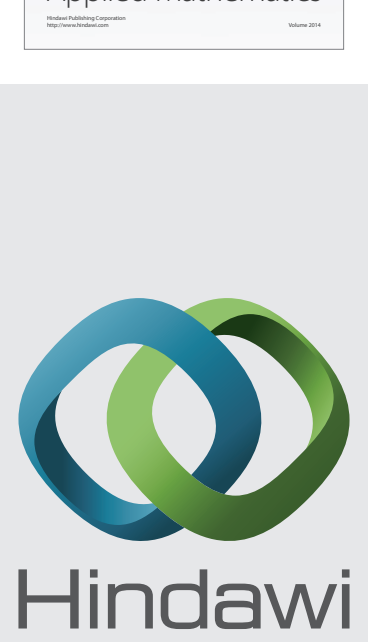

Submit your manuscripts at http://www.hindawi.com
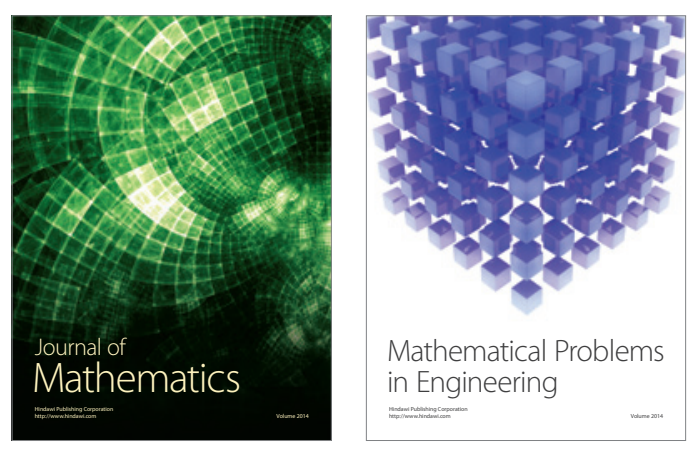

Mathematical Problems in Engineering
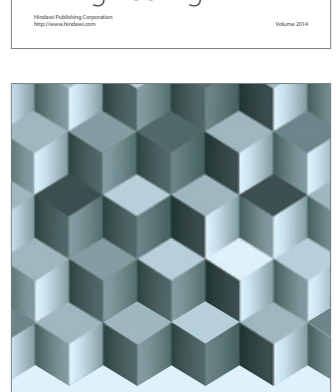

Journal of

Function Spaces
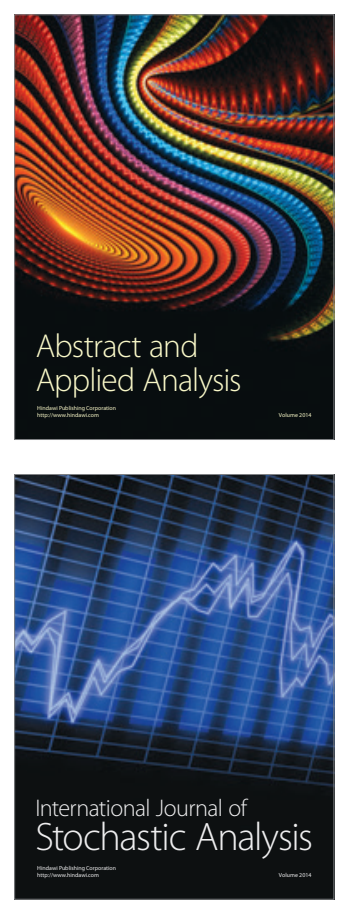

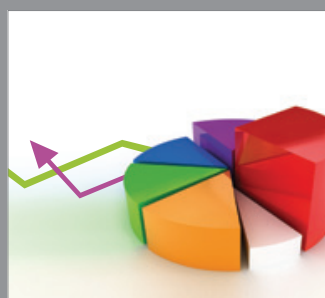

ournal of

Probability and Statistics

Promensencen
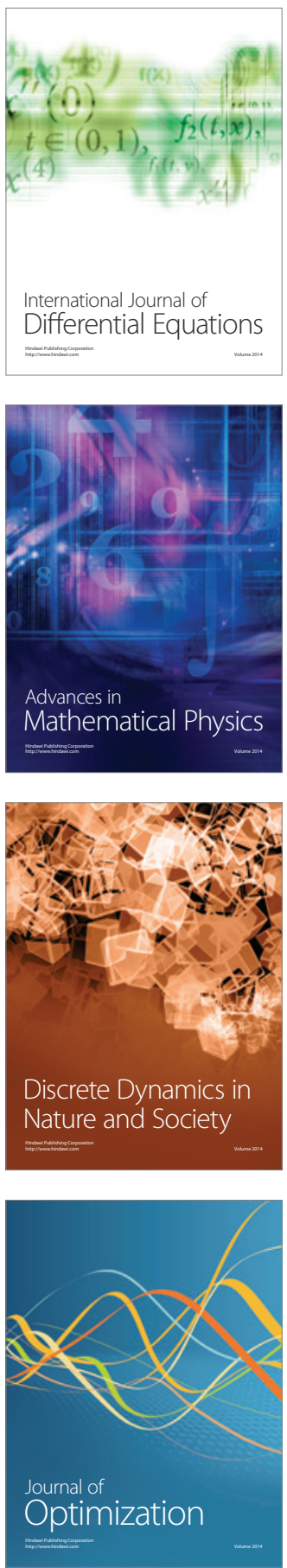